Supplement of Atmos. Chem. Phys., 21, 1015-1034, 2021

https://doi.org/10.5194/acp-21-1015-2021-supplement

(C) Author(s) 2021. This work is distributed under

the Creative Commons Attribution 4.0 License.

(c) (1)

Supplement of

\section{Atmospheric \\ Chemistry \\ and Physics}

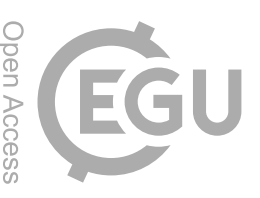

\title{
3D radiative heating of tropical upper tropospheric cloud systems derived from synergistic A-Train observations and machine learning
}

Claudia J. Stubenrauch et al.

Correspondence to: Claudia J. Stubenrauch (claudia.stubenrauch@1md.ipsl.fr)

The copyright of individual parts of the supplement might differ from the CC BY 4.0 License. 
Figures S1 and S2 present the MAE as a function of number of iterations (epochs), for cloudy scenes and for clear sky scenes,

15 respectively. The similarity in MAE between the validation and testing data means that there is no underfitting (the variables are not sufficient to predict the target) nor overfitting (the model is too detailed, with too many variables or the data base is not sufficiently large). The number of epochs to converge towards a minimum loss is relatively small: less than 60 for cloudy scenes (Figure S1) and less than 45 for clear sky scenes (Figure S2). Essentially, the MAE decreases considerably only within the first 10 (5) epochs for cloudy (clear sky) scenes. The relatively small number of epochs necessary for convergence may be

20 explained by the large statistics we use for the training and the number of relevant variables for the prediction. The final choice of parameters corresponds to Npar40 / Npar41 in Figure S1, as the MAE is smallest and comparable with the ones of Npar41 / Npar42 and Npar42 / Npar43. For clear sky scenes, Figure S3 compares the evolution of MAE for models developed over ocean over land and over both. Figure S2 shows that predictions over ocean will be better than over land which can be explained by a better homogeneity. Figure S2 also shows that by using the atmospheric profiles with a better vertical resolution (20 layers instead of 10) does not improve the results.

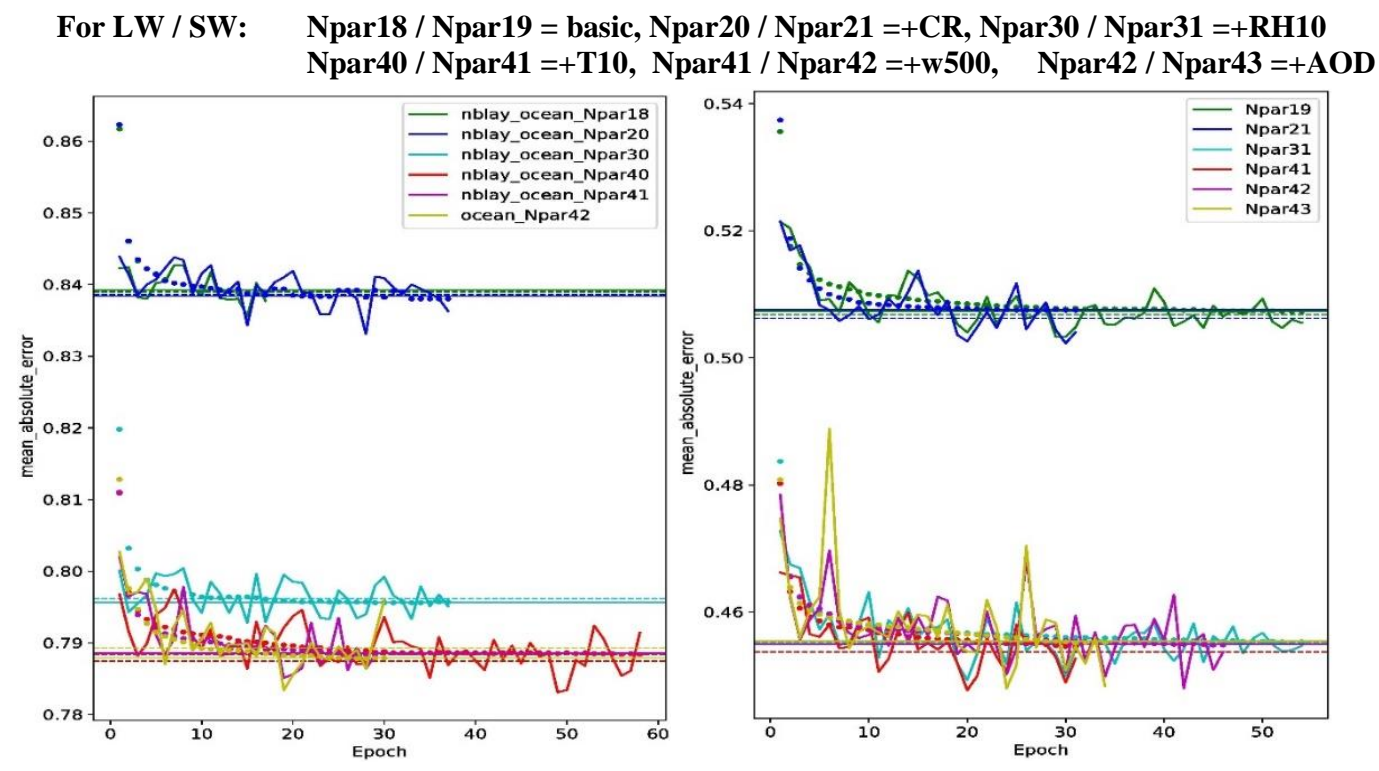

30 Figure S1: Sensitivity results concerning surface, atmospheric and cloud input parameters for the prediction of $L W$ (left) and SW (right) radiative heating rates of clouds over ocean: Mean absolute error (in K/day) of training (dots) and validation (lines) for the experiments 1-6 for $L W$ and 2 - 6 for SW, using the parameters listed in Table 1. 
For LW / SW: $\quad$ ocean_Npar35 / Npar35 = clear sky basic without cloud properties + clear sky fraction of CR + RH10 + T10, land_Npar38 / Npar36 = as for ocean, + 3 IR surface emissivities / + 1 surface albedo Npar39 / Npar37 = for land and ocean together, including land-ocean flag Npar60 / Npar58 = for land and ocean together, RH20 + T20 instead of +RH10+T10
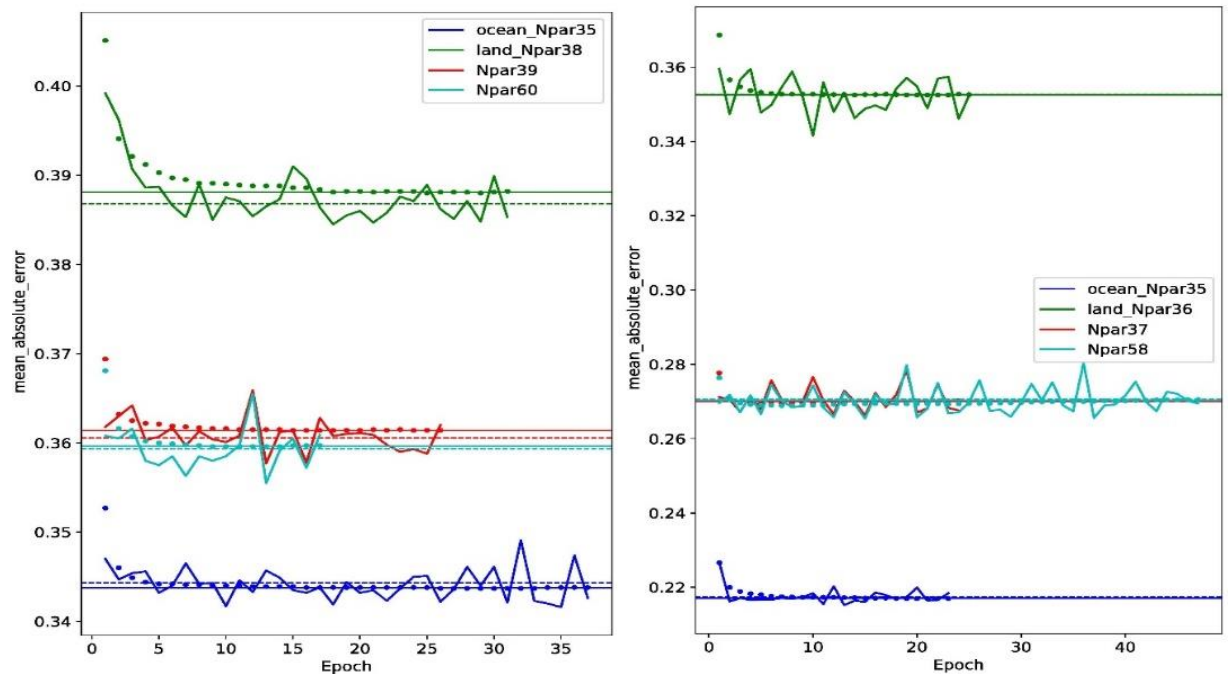

Figure S2: Sensitivity results for the prediction of $\mathrm{LW}$ (left) and SW (right) radiative heating rates of clear sky scenes as determined by CIRS: Mean absolute error (in K/day) of training (dots) and validation (lines): for the parameters listed in Table 1, excluding cloud properties and their uncertainties. 


\section{Sensitivity to the selection of scenes used for the training}

45 These sensitivity studies are dedicated to the scene types for which we develop the models:

i) all clouds over the whole tropical band (one model)

ii) all clouds separately over ocean and over land (two models)

iii) high-level clouds and mid- / low-level clouds individually, each separately over ocean and over land (four models)

iv) $\quad \mathrm{Cb}, \mathrm{Ci}$ / thin $\mathrm{Ci}$, mid- / low-level clouds individually, each separately over ocean and over land (six models) In addition, we develop models for clear sky i) over the whole tropical band (one model) and ii) separately over ocean and over land (two models). In general, a model trained over all scenes together soothes out differences between different cloud types and between ocean and land. Also scenes which are less frequent may have a smaller weight and may be therefore less represented than other scenes. Since we are interested in the study of the effect of UT cloud systems, we choose to use separate models. In particular, the modelling of $\mathrm{Cb}$ clouds is improved when exploiting a dedicated training for this cloud type, which represents about $7 \%$ of all clouds in the deep tropics (Stubenrauch et al., 2017).

To illustrate the effect of model aptness in dependence of training scenes, we compare in Figure S3 the difference between the predicted radiative HRs and those from CALIPSO-CloudSat over ocean for $\mathrm{Cb}$, cirrus and thin cirrus, in the LW and SW, respectively. Compared are models which were trained i) for all clouds over ocean and land together, ii) for all clouds over ocean, iii) for high-level clouds over ocean and iv) for $\mathrm{Cb}$ and for $\mathrm{Ci} /$ thin $\mathrm{Ci}$ over ocean. All results are quite similar, with the differences between mean predicted and 'observed' radiative HRs undulating well around $0 \mathrm{~K} /$ day. However, we observe an overestimation of the $\mathrm{LW}$ cooling above Cb clouds by nearly $1 \mathrm{~K} /$ day when all clouds together are used to develop one single model. The results improve for cirrus and thin cirrus when a dedicated model is developed for these cloud types. For the SW HRs it is not possible to determine the best performance among these four models. The SW heating in the upper part of $\mathrm{Cb}$ clouds is more difficult to predict, as for all four models the mean difference undulates around $0 \mathrm{~K} /$ day within $\pm 0.8 \mathrm{~K} /$ day between 100 and $200 \mathrm{hPa}$. Considering the radiative HR profiles shown in Figure S4 of the supplement, we find that the largest uncertainties for $\mathrm{Cb}$ clouds are around the maxima of LW cooling and SW heating. Furthermore, we observe that all models give very similar results, so that in the following we will mainly use the most specific scene models, leading to the application of eight models to reconstruct the radiative heating rate fields over the tropics.

70 We have also estimated the uncertainty related to the choice of scenes for the training after having applied these different ANN models to one month of AIRS data, over the whole tropical band (30N - 30S). Figure S5 presents for three atmospheric layers the difference between predicted LW HRs obtained from four models (clouds over ocean, clouds over land, clear sky over ocean, clear sky over land) and from two models (clouds and clear sky) and between predicted LW HRs obtained from all final eight models and from the four scene-dependent models. These differences, which give an indication of the uncertainty, lie 
75 generally within $0.25 \mathrm{~K} /$ day, with only a few regions of $0.45 \mathrm{~K} /$ day, keeping in mind that the most detailed scene distinction will give the better results.

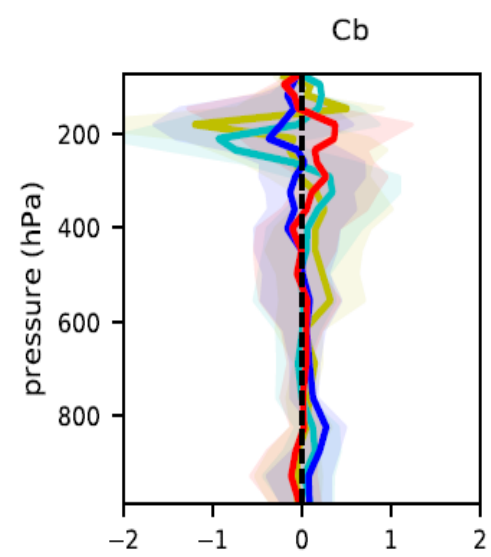

$\mathrm{Ci}$

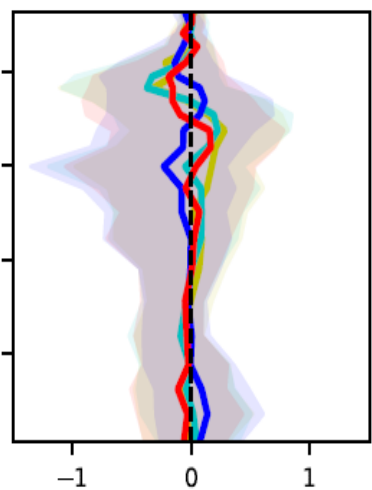

thin $\mathrm{Ci}$

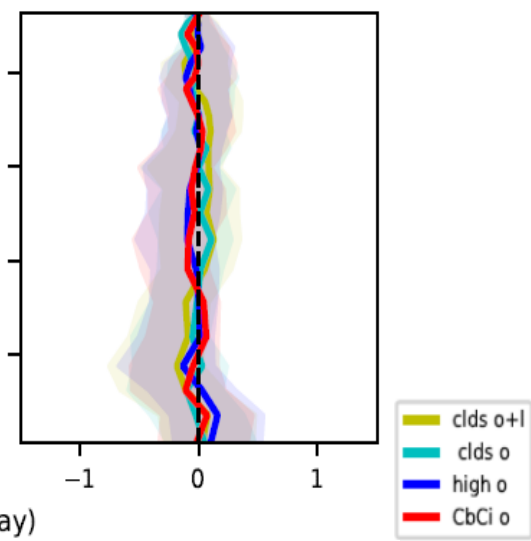

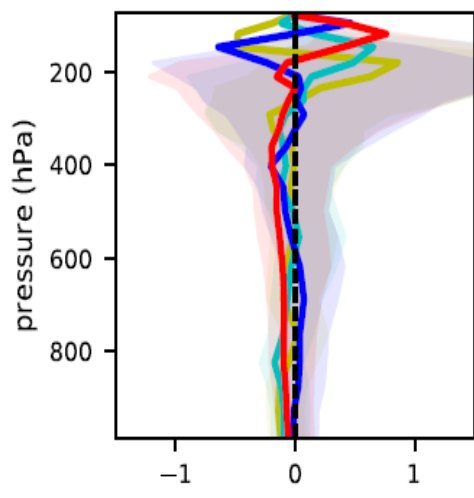

W HR (predicted-observed) (K/day)

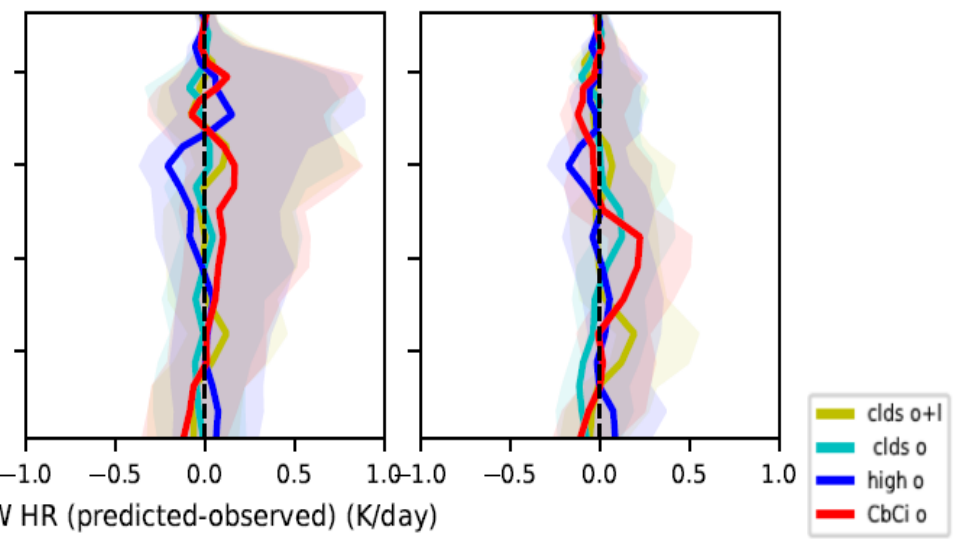

Figure S3: Sensitivity results concerning training over different scenes (high clouds over ocean, all clouds over ocean and all clouds over ocean and land) for the prediction of high-level cloud $L W$ radiative heating rates (above) and $S W$ radiative heating rates (below): difference between predicted and observed vertical profiles, separately for $\mathrm{Cb}$, Cirrus and thin Cirrus, as identified by AIRS-CIRS, over tropical ocean. 30\% and $70 \%$ quantiles of the distributions are also shown. 

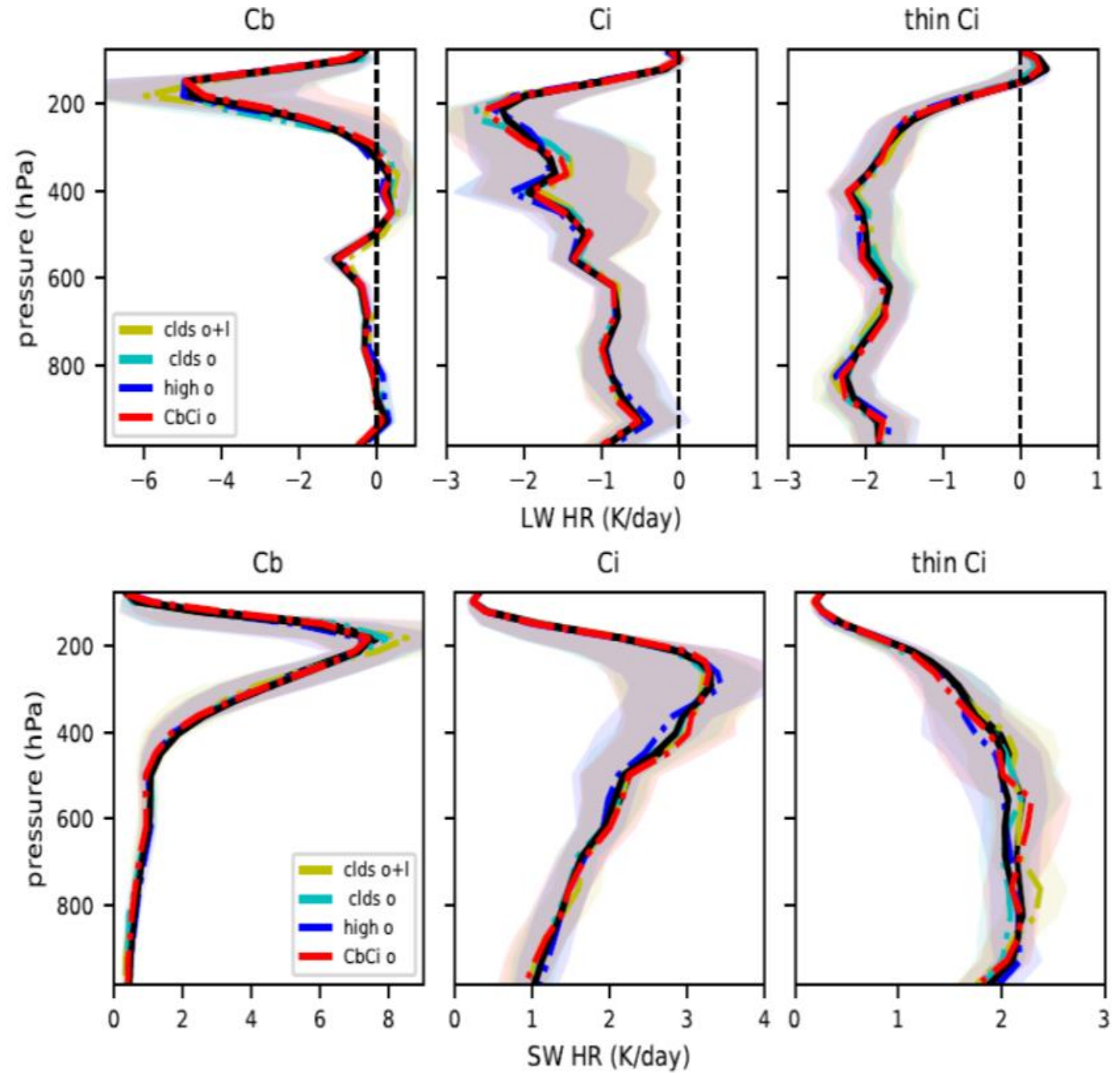

Figure S4: Sensitivity results concerning training over different scenes (high clouds over ocean, all clouds over ocean and all clouds over ocean and land) for the prediction of high-level cloud LW radiative heating rates (above) and SW radiative heating rates (below): predicted vertical profiles compared to those from CALIPSO-CloudSat (black lines), separately for $\mathrm{Cb}$, Cirrus and thin Cirrus, as identified by AIRS-CIRS, over tropical ocean. $30 \%$ and $70 \%$ quantiles of the distributions are also shown. 


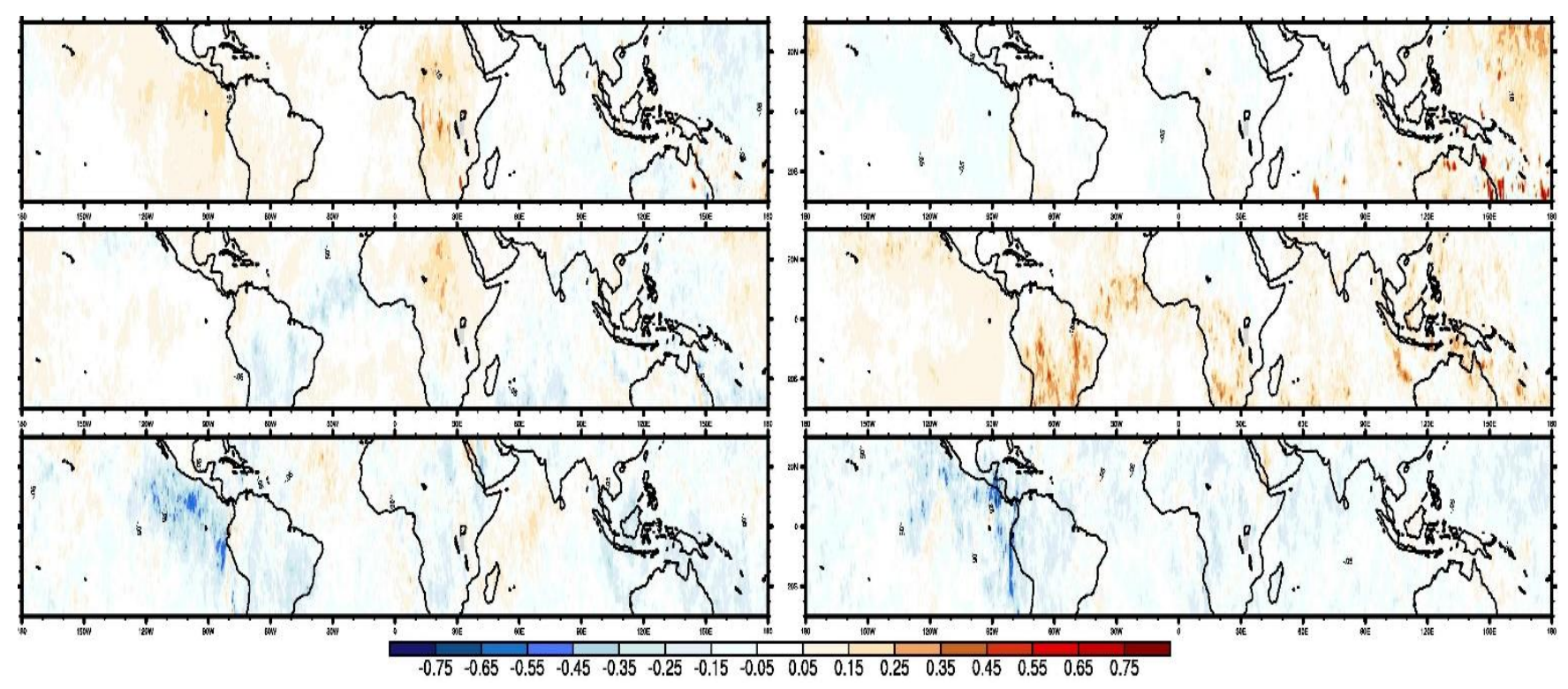

100 Figure S5: LW heating rate differences in 3 layers (106-131 hPa, 200-223 hPa, 525-585 hPa) between combination of (left) 4 models (clouds over ocean, clouds over land, clear sky over ocean, clear sky over land) and of 2 models (clouds and clear sky) and (right) 8 models and 4 models (clouds over ocean, clouds over land, clear sky over ocean, clear sky over land). 
Since the AIRS clear sky identification may also include subvisible cirrus as well as partly cloudy scenes within the AIRS

footprint, we estimated how much this affects the radiative HRs by comparing the FLXHR-lidar HRs for AIRS clear sky and for CALIPSO-CloudSat clear sky identification (no GEOPROF-lidar cloud layer within the footprint). Definitely, Figure S6 shows a slight positive bias in the clear sky LW heating near $100 \mathrm{hPa}$ of about 0.1 to $0.2 \mathrm{~K} /$ day due to subvisible cirrus, in particular during night, when the CALIPSO lidar better detects subvisible cirrus. The SW clear sky heating positive bias of the same order of magnitude between 200 and $800 \mathrm{hPa}$ and the cold LW clear sky heating negative bias around $900 \mathrm{hPa}$ are most

110 probably linked to contamination by partial cloudiness. As our present HR data are stored at a spatial resolution of $0.5^{\circ}$, we have identified another bias, which is due to the identification of clear sky at a spatial resolution of $0.5^{\circ}$. Clear sky HRs are sampled only over grid boxes with all AIRS footprints identified as clear sky. The broken lines in Figure S6 present the difference between the average HRs, deduced by machine learning and averaged over $0.5^{\circ}$ for cases with clear sky fraction equal to 1, and the average FLXHR-lidar HRs along the nadir tracks for the cases with CALIPSO-CloudSat clear sky

115 identification. This gives an estimation of the biases due to sampling at coarse spatial resolution and effects on the machine learning.

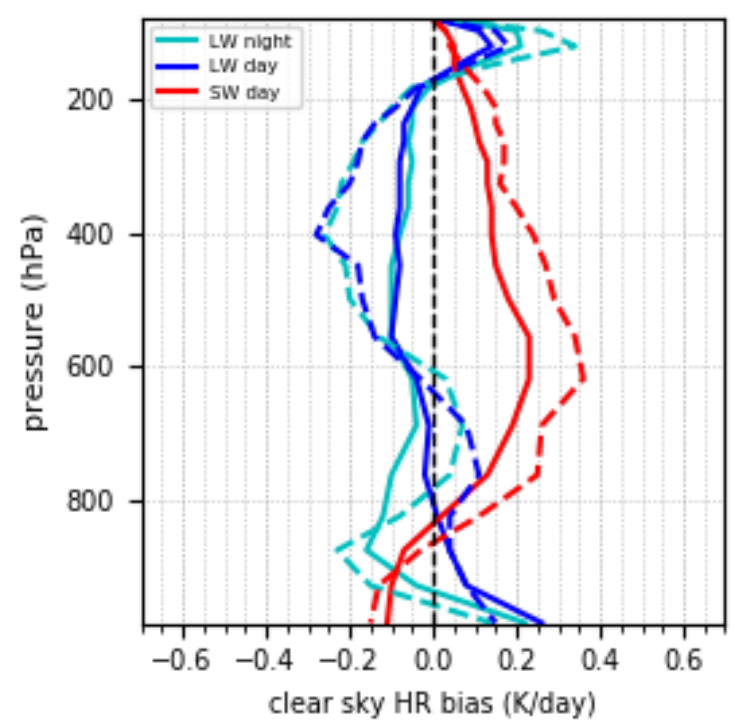

Figure S6: Bias in LW and SW heating rates due to uncertainties in CIRS clear sky identification (full line), given as mean difference of FLXHR radiative heating rates of clear sky identified as no CloudSat-lidar GEOPROF cloud layers and of 'clear sky - partly cloudy' identified by AIRS using the CIRS 'a posteriori' cloud detection, and in addition the effect of sampling for clear sky fraction 1 over $0.5^{\circ}$ (broken line), separately at nighttime (1:30 AM LT) and at daytime (1:30 PM LT). Statistics is over January 2008. 


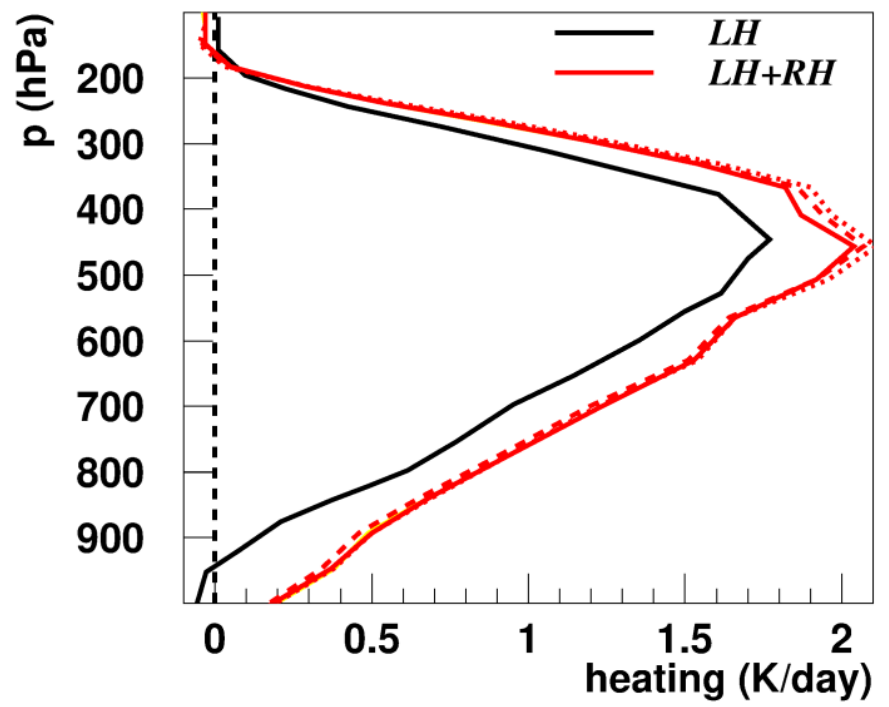

Figure S7: Tropical mean latent heating (black), digitized from Figure 9 of Li et al. (2013), and tropical mean diabatic 125 heating (red) as the sum of latent heating and net radiative heating (from Figure 5), including uncertainties due to cloud cover variation (dotted), LW HR variability between night and day (broken) and clear sky identification bias (only visible as slightly larger contribution near the surface and near the troposphere). 

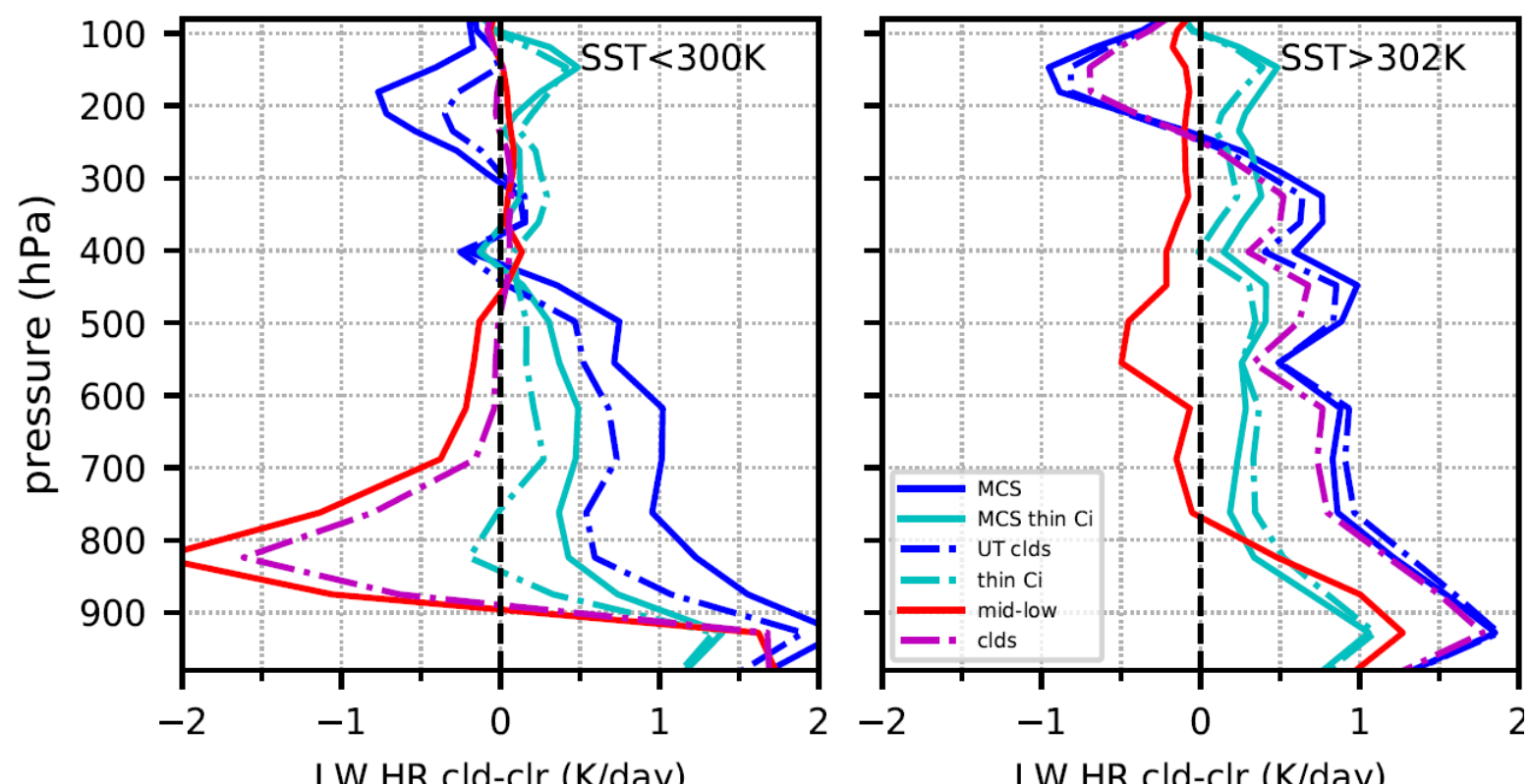

LW HR cld-clr (K/day)

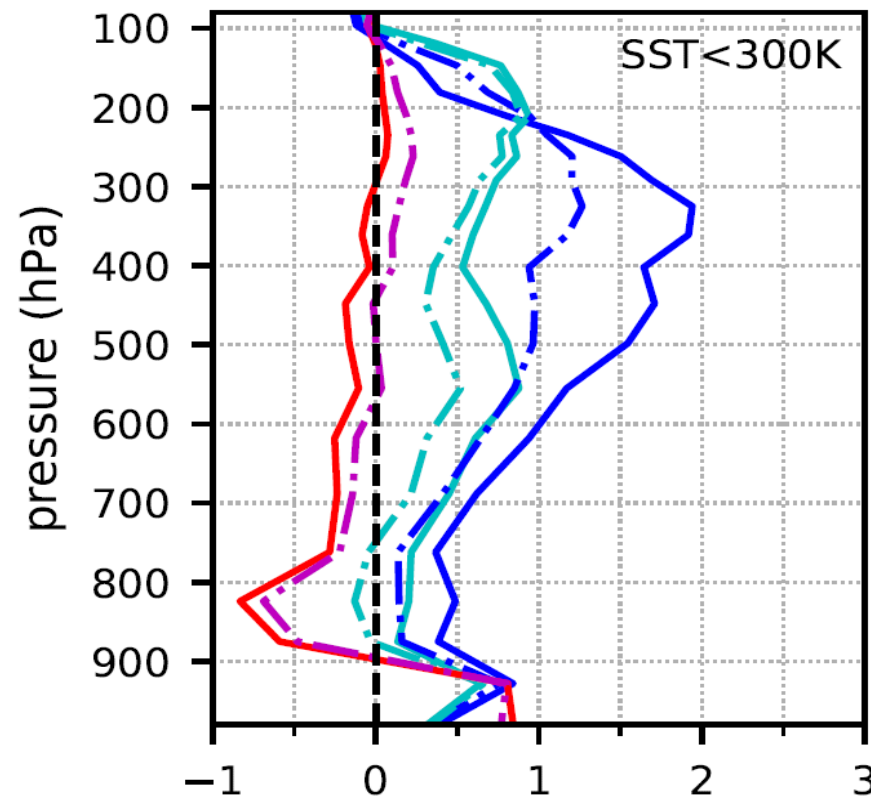

LW HR cld-clr (K/day)

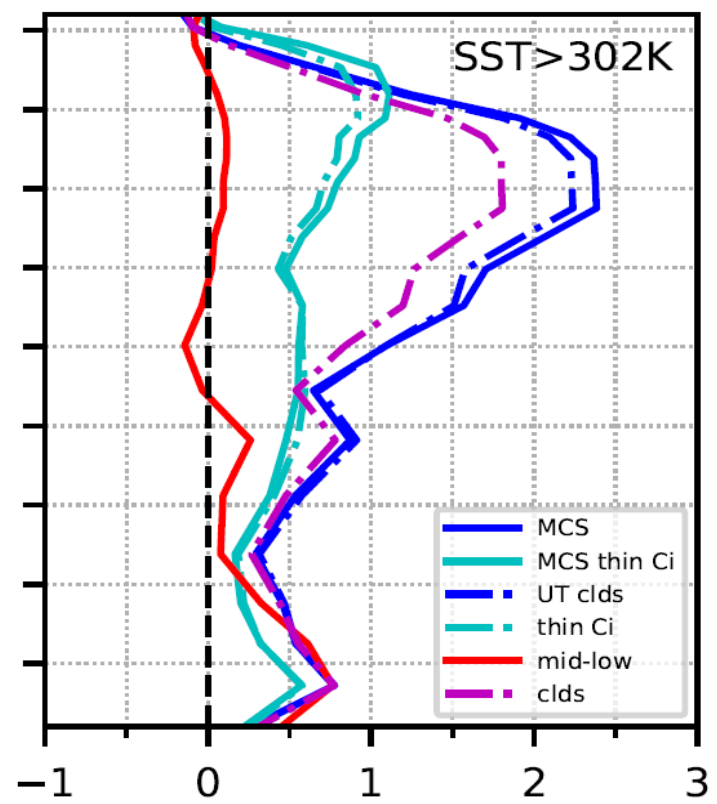

net HR cld-clr (K/day)

net HR cld-clr (K/day)

Figure S8: Tropical mean cloud net radiative heating effect (magenta) within the troposphere above ocean at 1:30 AM LT (top) and at 1:30 PM LT (bottom), as well as the separate effects of low- and mid-level clouds (red), all UT clouds (blue dash-dotted), thin cirrus (cyan dash dotted), MCS (blue full line) and thin cirrus associated with MCS (cyan full line), averaged over $15 \mathrm{~N}$ to $15 \mathrm{~S}$, when the specific cloud types are present. Left: regions with SST < $300 \mathrm{~K}$, right: regions with SST > $302 \mathrm{~K}$. 

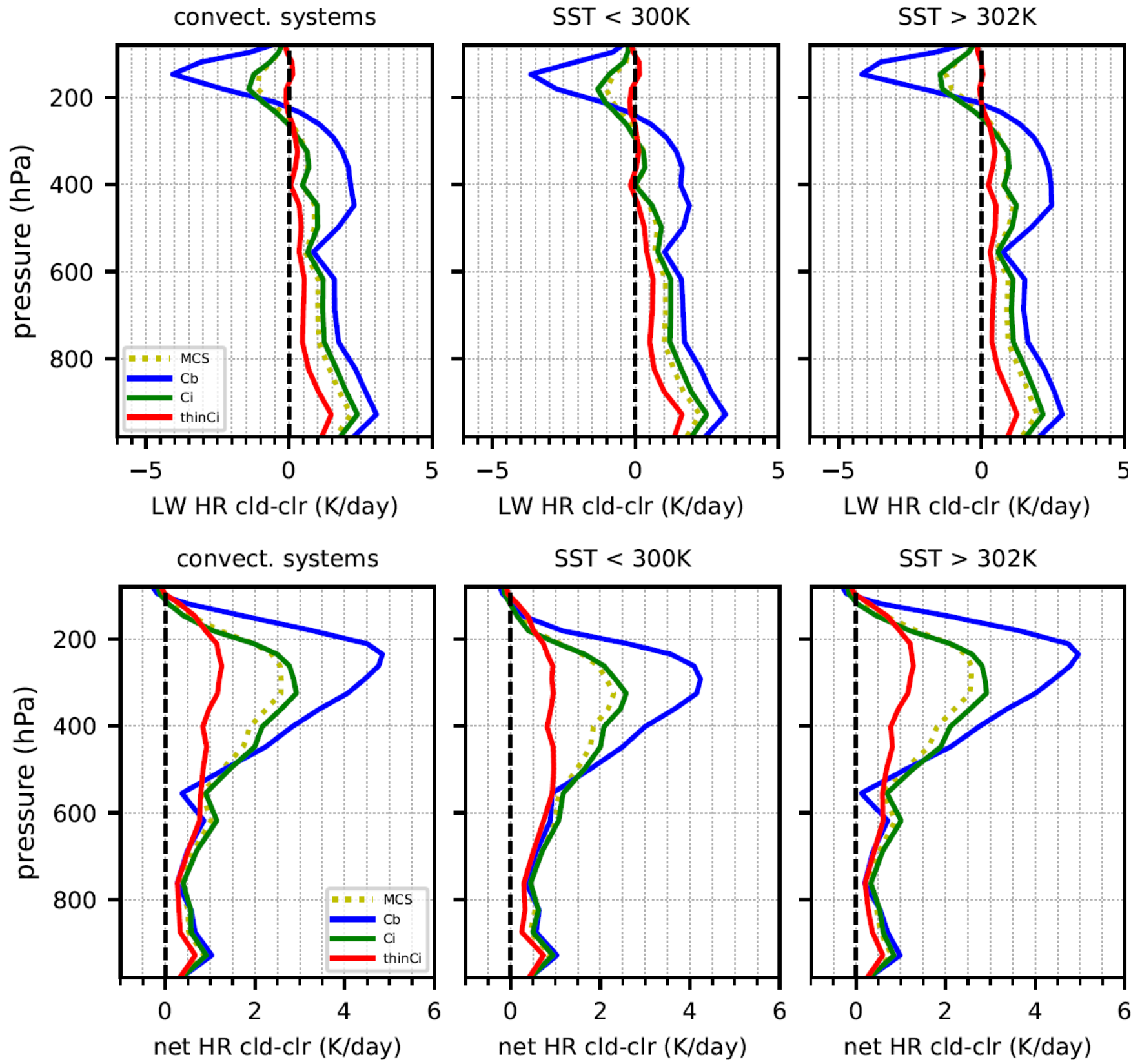

Figure S9: Mean net radiative heating effect of maritime MCSs, when present, and their convective cores (Cb), cirrus anvil (Ci) and surrounding thin cirrus (thinCi) at 1:30AM LT (top) and at 1:30 PM LT (bottom). Compared to MCSs over cool areas (SST < 300K) and to MCSs over warm areas (SST > 302K). Statistics of 15 years (2004-2018). 


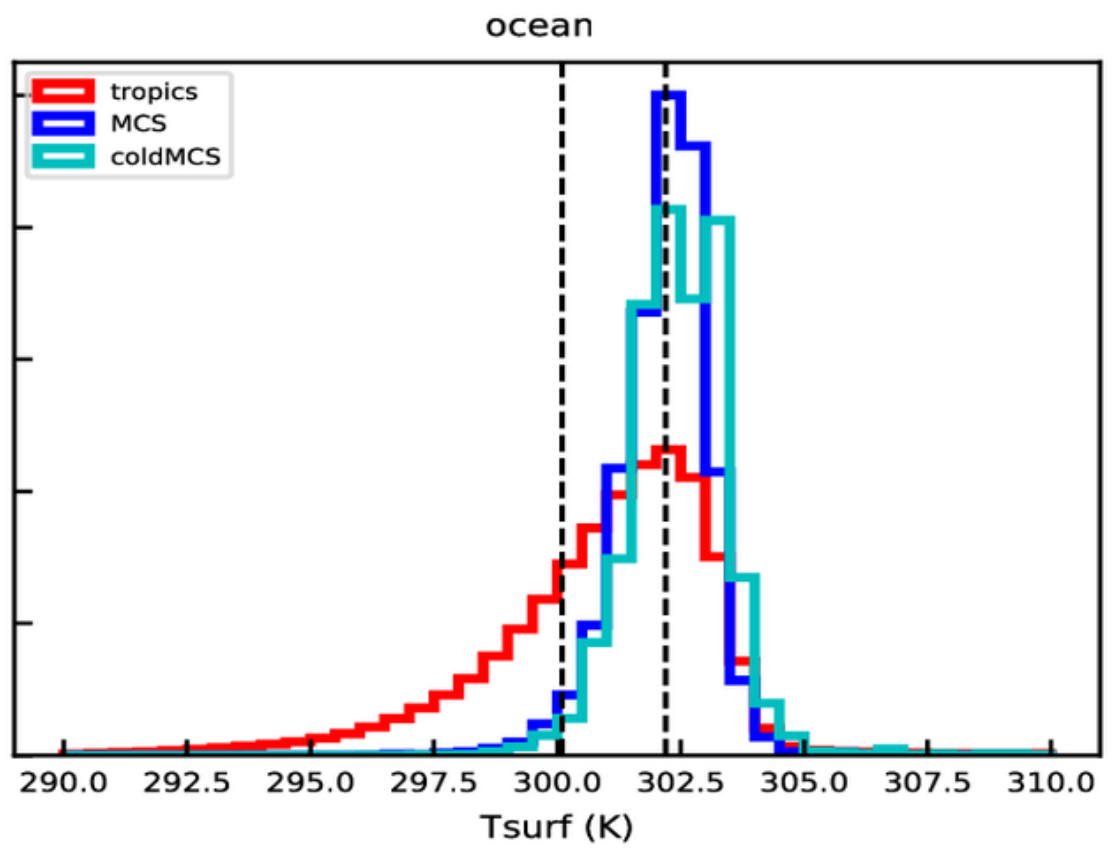

Figure S10: Normalized distributions of surface temperature (ERA-Interim) over tropical ocean (20N-20S) and of surface temperature underneath the opaque part (cloud emissivity $>0.9$ ) of all MCSs and of cold MCS (cloud 150 temperature of opaque part $<210 \mathrm{~K}$ ). The black lines correspond to the threshold temperatures for the 30\% coolest and $30 \%$ warmest surface temperatures, $300 \mathrm{~K}$ and $302 \mathrm{~K}$, respectively. 15 years statistics averaged over 1:30 AM and 1:30 PM LT. 

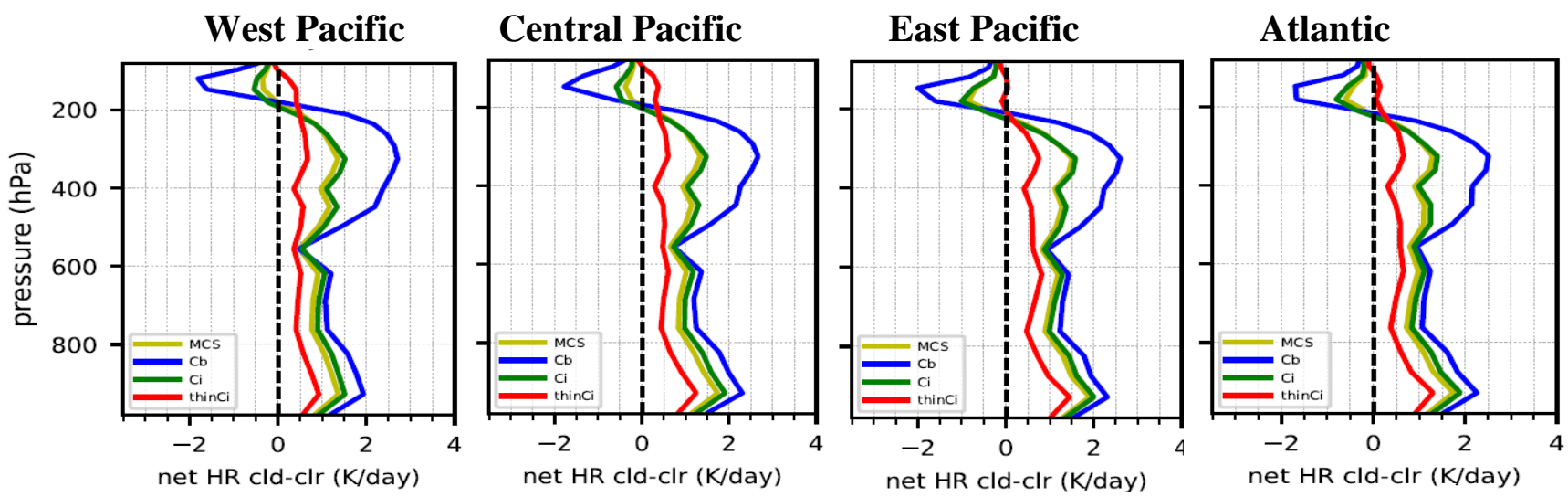

West Pacific

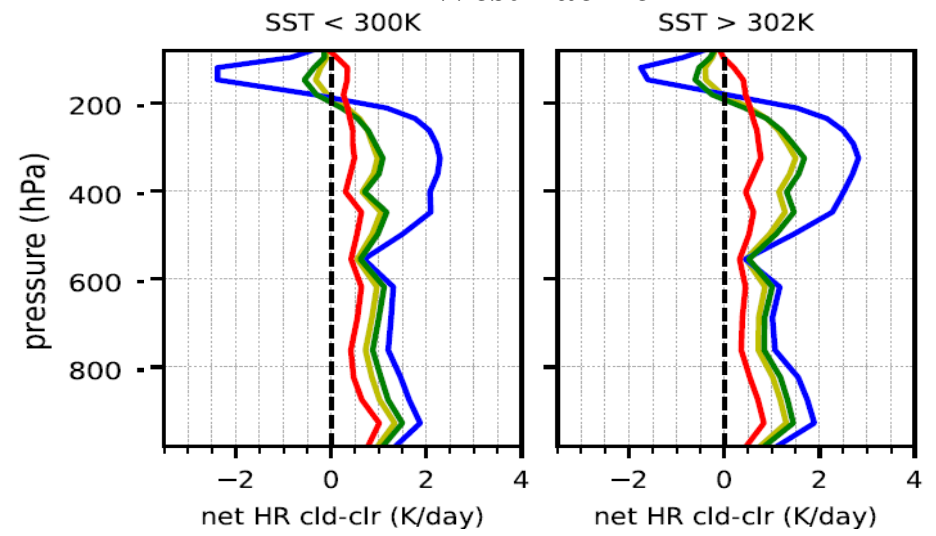

East Pacific
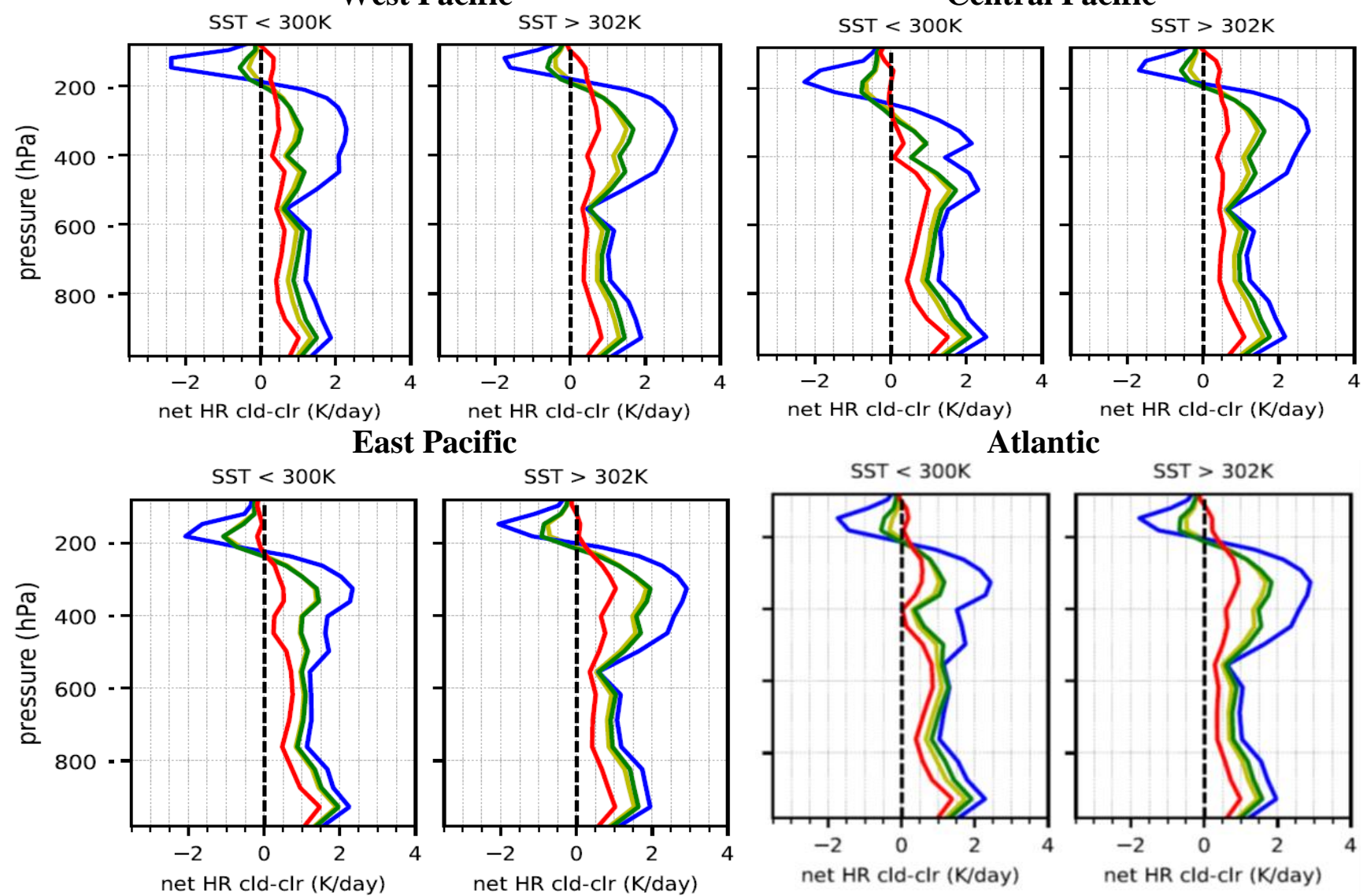

Atlantic

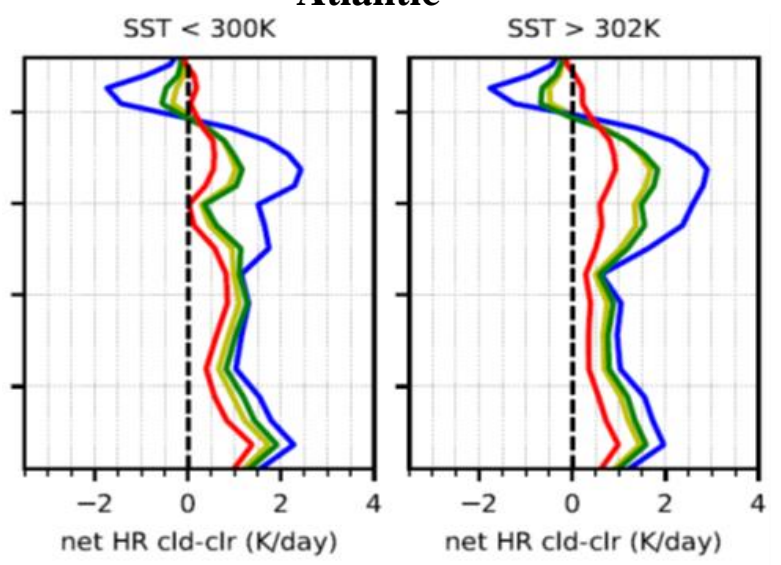

160 Figure S11: Mean 24-hr net radiative heating effect of maritime MCSs, when present, and their convective cores (Cb), cirrus anvil (Ci) and surrounding thin cirrus (thinCi). First panel from left to right: over four specific regions (West Pacific: 12N-12S and 130E-170E; Central Pacific: 10N-10S and 150W-180W; Eastern Pacific: 10N-10S and 100W-130W; Atlantic: 10N-10S and 5W-35W). Second and third panel: each of these regions separately over cool areas $(\mathrm{SST}<300 \mathrm{~K})$ and over warm areas (SST > 302K). Statistics of 15 years. 